\title{
Automated Classification of Brain MR Images Using Wavelet-Energy and Support Vector Machines
}

\author{
Guang-Shuai ZHANG, Qiong WANG, Chunmei \\ FENG \\ School of Computer Science and Technology \\ Nanjing Normal University \\ Nanjing, Jiangsu 210023, China
}

\author{
Genlin JI, Shuihua WANG, Yudong ZHANG* \\ Jiangsu Key Laboratory of 3D Printing Equipment and \\ Manufacturing \\ Nanjing, Jiangsu 210042, China
}

\author{
Elizabeth LEE \\ Department of Engineering Technology \\ Chattanooga State Community College \\ Chattanooga, TN 37406, USA
}

\begin{abstract}
It is of great importance to early detect abnormal brains, in order to save social resources. However, potential of wavelet decomposition is not fully explored and widely used. The wavelet-energy was a successful feature descriptor that achieved excellent performance in various applications; hence, we propose a wavelet-energy based new approach for automated classification of MR human brain images. The approach consisted of a three-stage system, including wavelet decomposition, energy extraction, and support vector machines. The results of proposed approach showed its performance was comparable with state-of-the-art algorithms. In addition, it provided a new means to detect features indicative of abnormal brains.
\end{abstract}

Keywords--MR images; MRI; classification.

\section{INTRODUCTION}

The problem of automatic classification of normal/pathological subjects based on brain MRI is of great importance in clinical medicine [1]. MRI is concerned with soft tissue anatomy and generates a large information set and details about the subject's brain condition [2]. There exists a large body of work on using brain MRI for automatic diagnosis [3, 4]. A promising approach for analyzing the obtained images is the wavelet transform which offers the capability of simultaneous feature localization in the time and frequency domains [5, $6]$.

By applying a wavelet transform to an image, four subbands are usually defined. They are the low-high, high-low, and high-high subbands which provide high-frequency coefficients for finer-scale image details representation, and the low-low subband whose low-frequency coefficients allow image approximation [7]. Wavelet transform have been applied in many areas of computer vision, of which image texture classification, and they have become popular for biomedical signal processing applications.

Scholars tend to use wavelets energy as inputs to a support vector machine to determine whether a MRI of the human brain is normal or abnormal [8-11]. The pathological images were those of brains suffering from Glioma, Huntington, Meningioma and Alzheimer's disease. The discrete wavelet transform was used to extract features from images and principal component analysis was employed reduce the number of features. SVM have attracted attention from the pattern recognition community. The proposed method, tested over MRI images, is developed with the aim of providing a computer-aided diagnosis tool that detects and selects the pathological brain images, and makes use of supervised learning techniques to classify new subjects, thus improving the accuracy of diagnosing pathological images in its early stage.

\section{METHODOLOGY}

\section{A. $2 D-D W T$}

The two-dimensional discrete wavelet transform (2D-DWT) decomposes an image into several sub-bands according to a recursive process $[12,13]$. The 1-level decomposition obtains two kinds of coefficients. One contains LH1, HL1, and HH1, which represent details of the original images $[14,15]$. The other is LL1 that corresponds to the approximation of original image [16]. The approximation LL1 is then decomposed into second-level approximation and detail images, and the process is repeated to achieve the desired level of resolution [17]. The obtained coefficients for the approximation and detail sub-band images are useful features for texture categorization. The 2D-DWT decomposes an image into spatial frequency components 
that describe the image texture. In practical, the coefficients are obtained by convolving the image with a bank of filters. Afterwards, selected features are extracted from the coefficients for further processing. Mathematically, the 2D-DWT of function $f(x)$ is defined by:

$$
\begin{aligned}
& f(x)=\sum_{i, j} c_{i, j} \psi_{i, j}(x) \\
& c_{i, j}=\int_{-\infty}^{+\infty} f(x) \psi_{i, j}(x)
\end{aligned}
$$

where $\psi_{i, j}(x)$ stands for the wavelet functions and cij are the DWT coefficients of $f(x)$.

\section{B. Wavelet-Energy}

In the first phase of the proposed work, two-dimensional discrete wavelet transformation is performed on the image. In our experiments, we fixed two decompositions level. After wavelet transformation is applied on the image, wavelet coefficients from the detail subbands of all the decomposition levels are used to formulate the local wavelet energy feature [5].

The wavelet energy in horizontal, vertical and diagonal directions at the i-level can be, respectively, defined as:

$$
\begin{aligned}
E_{i}^{h} & =\sum_{x=1}^{M} \sum_{y=1}^{N}\left(H_{i}(x, y)\right)^{2} \\
E_{i}^{v} & =\sum_{x=1}^{M} \sum_{y=1}^{N}\left(V_{i}(x, y)\right)^{2} \\
E_{i}^{d} & =\sum_{x=1}^{M} \sum_{y=1}^{N}\left(D_{i}(x, y)\right)^{2}
\end{aligned}
$$

These energies reflect the strength of the images' details in different direction at the i-level decomposition. So the feature vector, $\left(E_{i}^{h}, E_{i}^{v}, E_{i}^{d}\right)_{i=1,2 \ldots, k}$, where $\mathrm{k}$ is the total wavelet decomposition level, can describe the details features of facial expression effectively.

\section{C. $\quad S V M$}

The introduction of support vector machine (SVM) is a landmark of the field of machine learning. The advantages of SVMs include high accuracy, elegant mathematical tractability, and direct geometric interpretation [18]. Recently, multiple improved SVMs have grown rapidly, among which the kernel SVMs are the most popular and effective. Kernel SVMs have the following advantages [19]: (1) they work very well in practice and have been remarkably successful in such diverse fields as natural language categorization, bioinformatics and computer vision; (2) they have few tunable parameters; and (3) their training often involves convex quadratic optimization [20]. Hence, solutions are global and usually unique, thus avoiding the convergence to local minima exhibited by other statistical learning systems, such as neural networks.
Suppose some prescribed data points each belong to one of two classes, and the goal is to classify which class a new data point will be located in. Here a data point is viewed as a p-dimensional vector, and our task is to create a (p-1)-dimensional hyperplane. There are many possible hyperplanes that might classify the data successfully. One reasonable choice as the best hyperplane is the one that represents the largest separation, or margin, between the two classes, since we could expect better behavior in response to unseen data during training, i.e. better generalization performance. Therefore, we choose the hyperplane so that the distance from it to the nearest data point on each side is maximized. Figure 1 shows the geometric interpolation of linear SVMs, here H1, H2, H3 are three hyperplanes which can classify the two classes successfully, however, $\mathrm{H} 2$ and $\mathrm{H} 3$ does not have the largest margin, so they will not perform well to new test data. The $\mathrm{H} 1$ has the maximum margin to the support vectors (S11, S12, S13, S21, S22, and S23), so it is chosen as the best classification hyperplane.

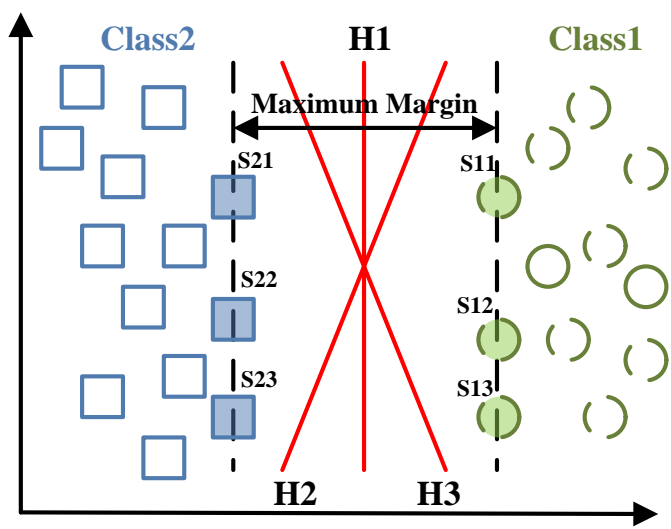

Figure 1. The geometric interpolation of linear SVMs (H denotes for the hyperplane, S denotes for the support vector)

Given a $p$-dimensional $N$-size training dataset of the form

$$
\left\{\left(x_{n}, y_{n}\right) \mid x_{n} \in R^{p}, y_{n} \in\{-1,+1\}\right\}, n=1, \ldots, N
$$

where $y_{n}$ is either -1 or 1 corresponds to the class 1 or 2 . Each $x_{n}$ is a p-dimensional vector. The maximum-margin hyperplane which divides class 1 from class 2 is the support vector machine we want. Considering that any hyperplane can be written in the form of

$$
\mathbf{w x}-b=0
$$

We want to choose the $\mathrm{W}$ and $\mathrm{b}$ to maximize the margin between the two parallel hyperplanes as large as possible while still separating the data. So we define the two parallel hyperplanes by the equations as

$$
\mathbf{w} \mathbf{x}-b= \pm 1
$$

Therefore, the task can be transformed to an optimization problem. That is, we want to maximize the distance between the two parallel hyperplanes, subject to prevent data falling into the margin. Using simple mathematical knowledge, the problem can be finalized as 


$$
\begin{aligned}
& \min _{\mathbf{w}, b}\|\mathbf{w}\| \\
& \text { s.t. } y_{n}\left(\mathbf{w} x_{n}-b\right) \geq 1, n=1, \ldots, N
\end{aligned}
$$

In practical situations the $\|\mathbf{w}\|$ is usually be replace by

$$
\begin{aligned}
& \min _{\mathbf{w}, b} \frac{1}{2}\|\mathbf{w}\|^{2} \\
& \text { s.t. } y_{n}\left(\mathbf{w} x_{n}-b\right) \geq 1, n=1, \ldots, N
\end{aligned}
$$

The reason leans upon the fact that $\|\mathrm{w}\|$ is involved to a square root calculation. After it is superseded with formula (10), the solution will not change, but the problem is altered into a quadratic programming optimization that is easy to solve by using Lagrange multipliers and standard quadratic programming techniques and programs.

\section{Implementation}

The proposed method is a combination of three successful components: WT, Energy Extraction, and SVM. The images belong to the whole brain atlas, where the brain image datasets are acquired using several imaging technologies.

Its flowchart is listed in follows. The proposed methodology of classifying MR images of human brain is shown in Figure 2. The method uses the steps of wavelet-transform, feature extraction, feature selection and classification.

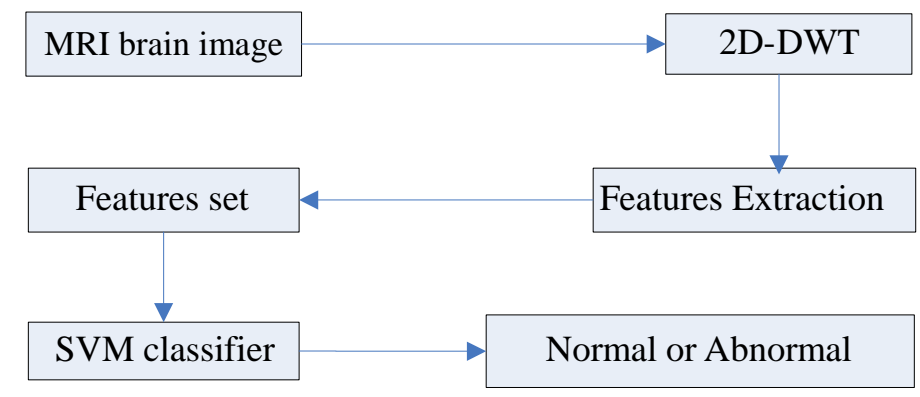

Figure 2. Flow diagram of the approach

\section{EXPERIMENTS}

\section{E. Data Acquirement}

We have tested our classification algorithm for several MR images, some of which belong to normal brain and others belong to pathological brain "Figure 3". All these normal and pathological images are axial, T2-weighted, MR images of $256 \times 256$ sizes. For each image available, the location of the image in the whole brain dataset is shown in the side view. For our case study, we have considered a total of 66 images (18 belonging to normal brains and 48 belonging to pathological brains, suffering from a low-grade glioma, meningioma, bronchogenic carcinoma, Glioblastoma, Sarcoma and Grade IV tumors) in several brain locations.

\section{F. Classification Comparison}

TABLE I CLASSIFICATION COMPARISON

\begin{tabular}{ll}
\hline Measure & Definition \\
\hline Accuracy & $(T P+T N) /(T P+T N+F P+F N)$ \\
Sensitivity & $T P /(T P+F N)$ \\
Specificity & $T N /(T N+F P)$ \\
\hline
\end{tabular}

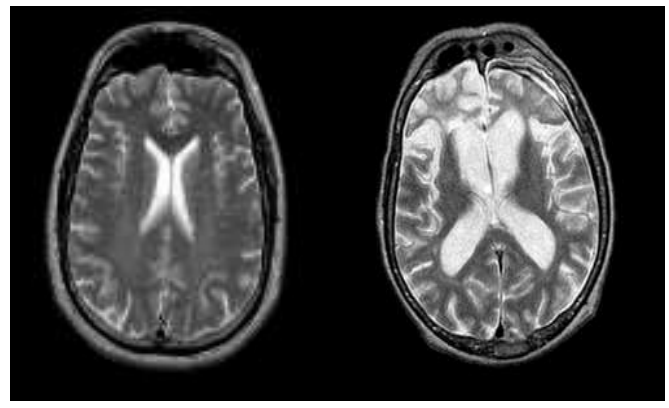

(a)

(b)

Figure 3. (a) A sample MR image of normal brain, (b) A sample MR image of abnormal brain

We compared the proposed wavelet-entropy with other reported features: including DWT+PCA+SVM [21], BPNN [22], RBFNN [22], and PSO-KSVM [22]. The evaluation measures are chosen as accuracy, sensitivity, and specificity shown in Table .

TABLE II CLASSIFICATION COMPARISON

\begin{tabular}{llll}
\hline Method & Accuracy & Sensitivity & Specificity \\
\hline DWT+PCA+SVM [21] & $95 \%$ & $96.42 \%$ & $85 \%$ \\
BPNN [22] & $86.22 \%$ & $88.00 \%$ & $56.00 \%$ \\
\hline RBFNN [22] & $91.33 \%$ & $92.47 \%$ & $72 \%$ \\
Wavelet-Energy+SVM & $82.69 \%$ & $78.05 \%$ & $\mathbf{1 0 0 . 0 0 \%}$ \\
(Proposed) & & & \\
\hline
\end{tabular}

Results in Table report that the accuracy, sensitivity, and specificity of the proposed wavelet-energy are $82.69 \%$, $78.05 \%$, and $100.00 \%$, respectively. Although the accuracy and sensitivity of the proposed method are slightly lower than latest methods [21,22], the specificity of the proposed method is perfect as high as $100 \%$, which 
is a substantial improvement to other methods. This suggested the wavelet-energy is extremely efficient in increasing the specificity of classification.

The disadvantage of the proposed method lies in its inefficiency of accuracy and sensitivity. The reason may attribute to following points: (i) decomposition level is too small to capture the discriminant feature; (ii) energy is not a good descriptor of brain MR image. However, considering its excellent performance of specificity, our method is competitive with latest methods.

\section{CONCLUSIONS AND FUTURE RESEARCH}

The contributions of this paper lie in following aspects: (i) A new approach for automatic classification of MR Images as normal or abnormal using WT, Wavelet-Energy and SVM classifier is proposed. (ii) Our experiments demonstrate the proposed classifier reaches a specificity of $100 \%$. (iii) Wavelet-Energy is an important and effective feature for MR brain image classification.

In this study, a new approach for automatic classification of MR Images as normal or abnormal using Wavelet-Energy and SVM is proposed. The results show that the proposed method gives comparable results with latest methods presented in the literature, but the proposed approach gives a specificity of $100 \%$. It suggests that our three-step algorithm is a promising for image classification in a medical imaging application. This automated analysis system, which requires much lighter computational time, could be further used for classification of image with different pathological condition, types and disease status.

In the future, we may focus on following regards: (i) we try to increase the decomposition level of 2D-DWT, in order to test whether higher level can lead to better classification performance. (ii) we may replace wavelet-energy with more efficient descriptors. (iii) Some advanced pattern recognition techniques may be used, such as RBF-NN [23] and deep learning [24].

\section{ACKNOWLEDGMENT}

This work was supported by NSFC (No. 610011024), Program of Natural Science Research of Jiangsu Higher Education Institutions of China (No. 14KJB520021), Jiangsu Key Laboratory of 3D Printing Equipment and Manufacturing (BM2013006), and Nanjing Normal University Research Foundation for Talented Scholars (No. 2013119XGQ0061).

\section{CONFLICT OF INTEREST}

We have no conflicts of interest to disclose with regard to the subject matter of this paper.

\section{REFERENCES}

[1] Zhang, Y., et al., "Energy Preserved Sampling for Compressed Sensing MRI," Computational and Mathematical Methods in Medicine, Vol. 2014, No. pp. 12, 2014.

[2] Kumari, P. V., K. Thanushkodi, and Ieee, "A Secure Fast 2D-Discrete Fractional Fourier Transform Based Medical Image Compression Using Hybrid Encoding Technique," 2013 International Conference on Current Trends in Engineering and Technology (Icctet), No. pp. 1-7, 2013.

[3] Goh, S., et al., "Mitochondrial dysfunction as a neurobiological subtype of autism spectrum disorder: Evidence from brain imaging," JAMA Psychiatry, Vol. 71, No. 6, pp. 665-671, 2014.

[4] Zhang, Y., et al., "Exponential wavelet iterative shrinkage thresholding algorithm with random shift for compressed sensing magnetic resonance imaging," IEEJ Transactions on Electrical and Electronic Engineering, Vol. 10, No. 1, pp. 116-117, 2015.

[5] Lee, S. H., et al., "Diagnostic method for insulated power cables based on wavelet energy," Ieice Electronics Express, Vol. 10, No. 12, pp. 2013.

[6] Dong, Z., et al., "Improving the spectral resolution and spectral fitting of $1 \mathrm{H}$ MRSI data from human calf muscle by the SPREAD technique," NMR in Biomedicine, Vol. 27, No. 11, pp. 1325-1332, 2014.

[7] Iqbal, A. and V. Jeoti, "A Novel Wavelet-Galerkin Method for Modeling Radio Wave Propagation in Tropospheric Ducts," Progress In Electromagnetics Research B, Vol. 36, No. pp. 35-52, 2012.

[8] Zhang, Y., S. Wang, and Z. Dong, "Classification of Alzheimer Disease Based on Structural Magnetic Resonance Imaging by Kernel Support Vector Machine Decision Tree," Progress In Electromagnetics Research, Vol. 144, No. pp. 171-184, 2014.

[9] El-Dahshan, E. S. A., et al., "Computer-aided diagnosis of human brain tumor through MRI: A survey and a new algorithm," Expert Systems with Applications, Vol. 41, No. 11, pp. 5526-5545, Sep 2014.

[10]Das, S., M. Chowdhury, and M. K. Kundu, "BRAIN MR IMAGE CLASSIFICATION USING MULTISCALE GEOMETRIC ANALYSIS OF RIPPLET," Progress in Electromagnetics Research-Pier, Vol. 137, No. pp. 1-17, 2013.

[11] Guerquin-Kern, M., et al., "A Fast Wavelet-Based Reconstruction Method for Magnetic Resonance Imaging," Medical Imaging, IEEE Transactions on, Vol. 30, No. 9, pp. 1649-1660, 2011.

[12]Nanthagopal, A. P. and R. S. Rajamony, "Classification of benign and malignant brain tumor CT images using wavelet texture parameters and neural network classifier," Journal of Visualization, Vol. 16, No. 1, pp. 19-28, 2013.

[13]Zhang, Y., et al., "Genetic Pattern Search and Its Application to Brain Image Classification," Mathematical Problems in Engineering, Vol. 2013, No. pp. 8, 2013.

[14]Jero, S. E., P. Ramu, and S. Ramakrishnan, "Discrete Wavelet Transform and Singular Value Decomposition Based ECG Steganography for Secured Patient Information Transmission," Journal of Medical Systems, Vol. 38, No. 10, pp. Oct 2014.

[15]Fang, L., L. Wu, and Y. Zhang, "A Novel Demodulation System Based on Continuous Wavelet Transform," Mathematical Problems in Engineering, Vol. 2015, No. pp. 9, 2015.

[16] Gandhi, T. K., et al., "Discrete harmony search based expert model for epileptic seizure detection in electroencephalography," Expert Systems with Applications, Vol. 39, No. 4, pp. 4055-4062, 2012.

[17]Rajendra Acharya, U., et al., "Use of principal component analysis for automatic classification of epileptic EEG activities in wavelet framework," Expert Systems with Applications, Vol. 39, No. 10, pp. 9072-9078, 2012.

[18] Martiskainen, P., et al., "Cow behaviour pattern recognition using a three-dimensional accelerometer and support vector machines," Applied Animal Behaviour Science, Vol. 119, No. 1-2, pp. 32-38, 2009.

[19]Bermejo, S., B. Monegal, and J. Cabestany, "Fish age categorization from otolith images using multi-class support vector machines," Fisheries Research, Vol. 84, No. 2, pp. 247-253, 2007.

[20]Muniz, A. M. S., et al., "Comparison among probabilistic neural network, support vector machine and logistic regression for evaluating the effect of subthalamic stimulation in Parkinson disease on ground reaction force during gait," Journal of Biomechanics, Vol. 43, No. 4, pp. 720-726, 2010.

[21]Zhang, Y. and L. Wu, "An Mr Brain Images Classifier via Principal Component Analysis and Kernel Support Vector Machine," Progress In Electromagnetics Research, Vol. 130, No. pp. 369-388, 2012.

[22]Zhang, Y., et al., "An MR Brain Images Classifier System via Particle Swarm Optimization and Kernel Support Vector Machine," The Scientific World Journal, Vol. 2013, No. pp. 9, 2013.

[23] Guo, D. L., et al., "Improved Radio Frequency Identification Indoor Localization Method via Radial Basis Function Neural Network," Mathematical Problems in Engineering, No. pp. 2014.

[24]Charalampous, K. and A. Gasteratos, "A tensor-based deep learning framework," Image and Vision Computing, Vol. 32, No. 11, pp. 916-929, Nov 2014. 